\title{
Reduction of Coincident Photomultiplier Noise Relevant to Astroparticle Physics Experiments
}

\author{
M. Robinson*, P. K. Lightfoot, M. J. Carson, V. A. Kudryavtsev, \\ N. J. C. Spooner \\ Department of Physics and Astronomy, University of Sheffield, Hicks \\ Building, Hounsfield Road, Sheffield S3 7RH, UK
}

\begin{abstract}
In low background and low threshold particle astrophysics experiments using observation of Cherenkov or scintillation light it is common to use pairs or arrays of photomultipliers operated in coincidence. In such circumstances, for instance in dark matter and neutrino experiments, unexpected PMT noise events have been observed, probably arising from generation of light from one PMT being detected by one or more other PMTs. We describe here experimental investigation of such coincident noise events and development of new techniques to remove them using novel pulse shape discrimination procedures. When applied to data from a low background $\mathrm{NaI}$ detector with facing PMTs the new procedures are found to improve noise rejection by a factor of 20 over conventional techniques, with significantly reduced loss of signal events.
\end{abstract}

Key words: Photomultiplier noise, Dark matter, Scintillators PACS: 85.60.H 95.35 29.40.Mc

Corresponding author: Matthew Robinson, Department of Physics and Astronomy, University of Sheffield, Hicks Building, Hounsfield Road, Sheffield S3 7RH, Tel: +44 (0)114 2223553, Fax: +44 (0)114 2728079, E-mail: matthew.robinson@sheffield.ac.uk

*Corresponding author: Matthew Robinson E-mail: matthew.robinson@sheffield.ac.uk 


\section{Introduction}

Photomultiplier noise can significantly limit the sensitivity of low background and low threshold astroparticle physics experiments searching for rare events such as neutrinos or WIMP dark matter using Cherenkov or scintillator technology. The drive for low threshold, which determines, for instance, the minimum energy of neutrino that can be detected in an astrophysical neutrino detector, requires detection of the lowest possible number of photons in the target medium. This translates to design geometries that use many photomultipliers to view the medium, operated in coincidence. Such designs provide the necessary good light collection but furthermore, because of the coincidence operation, provide an effective means of suppressing certain types of well known PMT (Photomultiplier Tubes) noise, in particular single-photoelectron (SPE) noise and after-pulses. For these types of noise there is no causal relation between noise generated in a given tube and that in any other tube.

However, operation of these designs in which PMTs are effectively light coupled together, makes them vulnerable to other forms of PMT noise, in particular to effects often ascribed to "dynode glow" [1, 2]. This is believed to be due to light emitted from the dynode stack under electron bombardment being scattered and reflected back to the photocathode. This can produce noise in the host tube but additionally the photons produced can in principle also be observed by all light-coupled tubes. Such a coincident event is not suppressed by the coincidence operation and may mimic a signal event. Significant phenomena of this sort have been observed in the SNO detector, where they have been termed "flashers". These are seen at $\sim 1000$ /day above an 18 hit threshold comprising relatively prompt flashes of light attributed to discharges from the dynodes [3]. In dark matter detectors based on NaI, liquid Xe and other scintillators where high sensitivity and low background is needed, a likely related class of events sometimes termed "step" events is seen [4, 5]. The drive for lower thresholds and lower backgrounds means that such unusual and rare events will become an increasingly significant source of systematic error in these and other planned PMT-based low background detectors.

In this paper we describe investigation of such coincident PMT noise events and the development of new techniques to identify and suppress these events. The work was undertaken using apparatus at the Boulby Underground Laboratory comprising a low background shielding castle within which can be mounted pairs of face-to-face low background photomultipliers with and without scintillator in various configurations (see sec 2). The apparatus used is based on that of the modules of the NAIAD (Sodium Iodide (NaI) Advanced Detector) experiment [6]. The NAIAD experiment is a direct

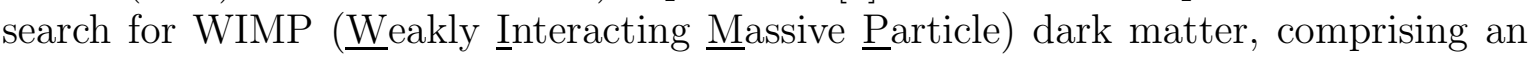
array of 8 shielded low background $\mathrm{NaI}(\mathrm{Tl})$ crystals viewed by pairs of photomultipliers 7. WIMP search experiments use various techniques to distinguish between nuclear recoil events, expected from WIMP interactions, and background electron recoil events. In the case of $\mathrm{NaI}(\mathrm{Tl})$ crystals, such as used in NAIAD, and several other experiments, this discrimination is achieved using pulse shape analysis to identify nuclear recoils 6. 7, 8, 9, 10, 11]. This is possible because in $\mathrm{NaI}(\mathrm{Tl})$, for instance, the scintillator

time constant for nuclear recoil events is shorter (typically 170-200 ns) than for electron recoils (typically 240-300 ns).

Dark matter limits are set using this technique by determining statistically the 
maximum number of recoil events that may be present in a dataset. However, as with many such low background experiments it is crucial to have sensitivity at the lowest possible energy, to probe the maximum range of initial particle energy or mass. For this reason the coincidence threshold is usually set low, for instance at approximately 1 $\mathrm{keV}$ in NAIAD, allowing a sensitivity to WIMP masses in principle below $100 \mathrm{GeV}$. In such experiments the rate of PMT noise events is greatly reduced by using coincidence counting which demands that each PMT should see a minimum signal for an event to be accepted. However, any coincident PMT noise remains and strategies to reduce this (rather than just the SPE or non-causal noise) then become of major importance.

In low background experiments using optically coupled PMTs such as this, some suppression of coincident noise can be achieved by the following two techniques: (a) additional pulse shape analysis of the events, and (b) asymmetry cuts. The former technique is based on the observation that the distribution of time constants of low energy PMT coincident noise events can be fitted to an exponential function. This has a faster characteristic mean decay time than that of most scintillator events (though slower than the normal PMT response time). This distribution can be fitted and then subtracted from the data, even though the distribution overlaps with the distribution of time constants for scintillation events. Figure 1 shows a typical low energy time constant distribution for $\mathrm{NaI}$ including this noise feature. The asymmetry cut technique is based mainly on comparing the characteristics of coincident events, such as amplitude, absolute start time of the pulse and pulse shape 6, 17, 12]. The assumption used is that coincident noise seen in the tubes is due to light emitted from one tube being seen by one or more other tubes, as suggested occurs in SNO and dark matter experiments [3. 5]. In this case, unlike the situation for genuine signal events, the characteristics are likely to be asymmetric, for instance the pulse in one PMT being of much larger amplitude than the other, or with faster rise time.

Although these techniques are useful they both have clear drawbacks. The coincident noise subtraction technique, although powerful, assumes that the exponential fit of short time constant events may be extrapolated to higher time constants, through the data events. This assumption introdues systematic errors into the results. Such a subtraction also necessarily entails the possible rejection of genuine events, requiring the introduction of an efficiency factor. The asymmetry cuts can not remove coincident noise events which give pulses in the tubes of similar characteristic. The asymmetry technique also requires operation in coincident mode, which may not always be possible or desirable. For dark matter searches any PMT noise events misidentified as nuclear recoil events reduce the effectiveness of pulse shape discrimination analysis.

Based on the drawbacks above there is a need to develop new techniques to reject coincident noise events with fit time constants comparable to signal events. We describe here new work in this area. Firstly, the apparatus used to acquire and characterise samples of low background coincident PMT noise data is outlined. This is followed by details of how the data were analysed and compared with data taken with similar configurations but with scintillator present. We then discuss the development of analysis techniques designed specifically to identify and reject coincident PMT noise. Finally, we will show comparisons between the effectiveness of the new procedures and more traditional techniques. 


\section{Experimental setup and data}

All PMT noise measurements were performed at the Boulby Underground Laboratory at $1070 \mathrm{~m}$ depth, using the apparatus shown in Figure 2. The set up comprises a shielding castle, incorporating $15 \mathrm{~cm}$ of low background lead, inside of which is a further $10 \mathrm{~cm}$ layer of copper shielding. The castle was supplied with an automatic source dropper to allow remote calibration of the scintillator using $\gamma$-ray sources $\left({ }^{57} \mathrm{Co}\right.$ and $\left.{ }^{60} \mathrm{Co}\right)$. A copper support structure allows 5 inch PMTs to be mounted face to face in a variety of configurations within the castle. EMI 93905 inch PMTs were used throughout. Four arrangements were used in the present work:

(i) PMTs optically coupled to each other through two $14 \mathrm{~cm}$ diameter cylindrical quartz light guides each $5 \mathrm{~cm}$ thick.

(ii) PMTs optically coupled directly with no intervening light guide.

(iii) PMTs coupled together but with the quartz lightguides separated and coupled through a $5 \mathrm{~cm}$ long $\mathrm{NaI}(\mathrm{Tl})$ crystal of $14 \mathrm{~cm}$ diameter.

(iv) PMTs coupled directly face to face but with an opaque barrier to stop direct light transmission between the tubes.

Data were collected using an in-house LabView based data aquisition system [5], the signals from each PMT being first passed through an integrating buffer. The integrated signals were fed to discriminator units set to trigger when signals exceed a threshold of $10 \mathrm{mV}$, equivalent to 4 photoelectrons. The logic signals from the discriminator units were fed to a coincidence unit which triggers a digital oscilloscope when signals arrive from both discriminators. When the oscilloscope was triggered, the integrated signals from both PMTs were digitised and stored, each digitisation point representing 10 ns elapsed time. These signals were then read through a GPIB (General Purpose Interface Bus) system into a computer which wrote the digitised pulses to a data file for later analysis.

Figure 3 shows an example gamma pulse from the system when configured with set-up (iii) with the light guides and $\mathrm{NaI}(\mathrm{Tl})$ crystal in place. Two integrated pulses, one from each tube, are seen, plus the summed pulse. In such integrated pulses single photoelectrons are observed as small steps in the pulse. A conventional pulse shape analysis, such as used in dark matter searches, is performed by fitting the summed integrated signal to an exponential function:

$$
f\left(A, t, t_{0}, \tau\right)=A\left(1-e^{\frac{\left(t_{0}-t\right)}{\tau}}\right)
$$

where $A$ is the amplitude of the integrated pulse, which is proportional to the number of photoelectrons, $t_{0}$ is the start time of the rise of the integrated pulse, and $\tau$ is the exponential fit time constant. Further details of this analysis are given in [6, 17.

A pulse such as shown in Figure 3 is most likely due to scintillation. Tests with the apparatus configured in an identical manner but without the crystal in place (set-up (i)) reveal, as shown by the example pulse in Figure 4, that coincident PMT noise pulses may lead to pulse shapes with similar characteristics. Tests without the light guides in place (set-up (ii)) demonstrated that similar events are still present and so confirmed that the quartz itself is not responsible for the events. Events of this kind would not be cut by conventional asymmetry cuts as the amplitudes and shapes in both tubes 
are similar. Identical runs but with an opaque card in place to block direct passage of light between the tubes (set-up (iv)) confirmed that in this case no coincident events are observed. All these results together confirm the suspicion that slow ( $>100 \mathrm{~ns}$ ) coincident noise events exist and that they are due to light generated in one tube being recorded by both tubes.

In accordance with $\mathrm{NaI}$ analysis procedures developed by the UKDM Collaboration 6. 17, 12, data recorded from the experiments here were binned in 2-d histograms of pulse height (energy) and fit time constant. Figure 5 (upper points) shows a typical extracted 1-d time constant distribution for the coincident noise events taken without scintillator for a pulse amplitude band equivalent to 4-6 keV (set-up (i)) with no cuts. With the scintillator in place calibration with ${ }^{57} \mathrm{Co}$ (122 keV gamma line) is used to determine the energy scale. Comparison with the results with no scintillator then allows an effective energy scale to be determined when no scintillator is present. For the tests with scintillator a ${ }^{60}$ Co source was used to generate low energy compton electron recoils via compton scattering in the crystal with a broad spectrum of energies. Since the rate of scintillation events in such calibration runs is much higher than that of any noise events the data may be used as a pure sample of scintillation events. By comparing noise events from data collected with no scintillator and scintillation events from data collected with no noise, with similar amplitude and time constant, it is possible to identify features which are much more common in the noise events than in scintillator events and thereby to identify noise events in real data. The rate fall off with increasing time constant seen in Figure 5 (upper plot), has the characteristic exponential form that is used as a basis for the conventinal noise subtraction technique [12. This form is seen clearly in the NaI data of Figure 1 at low time constant.

\section{Investigation and improved reduction of noise}

Typical asymmetry cuts used to reject noise from NAIAD data, reject events where the fit time constants of pulses from each PMT differ by 100 ns or more, or where the start times of the pulses differ by 100 ns or more, or where the energy associated with the pulses differs by $40 \%$ of the combined energy or more [5, 12]. Cuts based on these parameters represent the current best procedures but, as discussed above, do not adeqately deal with the coincident noise phenomena. To improve the situation and develop better rejection strategies the characteristics of noise pulses were examined more closely. This study revealed, in particular, that a large population of the observed smaller multi-photoelectron noise events when fitted to exponentials had very short time constants $(<100 \mathrm{~ns})$. Based on this, the assumption was made that the noise events observed with longer time constants are combinations of such "step" events each separated by a few tens of ns. These steps may have some causal relationship but are not the result of a true exponential decay as is the case for true scintillation pulses. Working from this assumption, various quantities relating to the quality of fits to exponentials were investigated, to determine which could be used to distinguish the multi-step noise events from scintillation events with a similar decay profile. Cuts defined based on these quantities have been termed "quality cuts". 
The most obvious quality cut arises from measuring the $\chi^{2}$ per degree of freedom:

$$
\chi^{2}=\sum_{i=n_{0}}^{n_{1}} \frac{\left(f\left(A, t_{i}, t_{0}, \tau\right)-D_{i}\right)^{2}}{\sigma_{i}^{2}},
$$

where $n_{0}$ is the first data point included, $n_{1}$ is the last data point included, $f\left(t_{i}, t_{0}, \tau\right)$ is the fit as described in Equation 1, $t_{i}$ is the time at digitisation point $i, D_{i}$ is the measured amplitude at that digitisation point and $\sigma_{i}$ is the uncertainty on that amplitude.

Unfortunately, although a least squares method is used to fit the pulses to an exponential, calculating the $\chi^{2}$ is not straightforward because the fit is performed on integrated pulses and the uncertainties on each digitised pulse point are not defined. To deal with this problem, a value for uncertainty is chosen for each pulse and applied to all digitised points within that pulse. This uncertainty is chosen to be proportional to the square root of the total number of photoelectrons the pulse represents and is normalised to yield an average $\chi^{2}$ per degree of freedom of 1.0 for scintillation events of all energies.

The rise of an integrated pulse represents the arrival of photons at the PMT, but the flat part of the pulse following the rise does not. Since no new information is associated with this part of the digitised pulse, tests were made with evaluation of the fit quality within limited time regions of the pulse. The typical scintillation time constant is 250-300 ns and it was found that calculation of $\chi^{2}$ per degree of freedom using the first $50 \mathrm{~ns}, 50$ to $100 \mathrm{~ns}$ or 100 to $500 \mathrm{~ns}$ of pulse data points give quantities which are useful in identifying noise events. The $\chi^{2}$ calculated using the full pulse was also found to be useful. Usually one or more of these particular quality parameters is typically larger for a given noise event than for similar scintillation events. However, no one parameter set appears sufficient alone to identify all noise events. Noise may be identified by some of the parameters but missed by others.

To address this, a further new quality cut technique was devised which we have called "steppiness". Steppiness $(\Gamma)$ provides a means to quantify the tendency of the digitised PMT coinicident noise pulse to be made up of multi-photoelectron steps. It is described by the following equations:

$$
\begin{gathered}
\Gamma=\frac{\sum_{i=1}^{N-n} \frac{\mu_{i}}{f^{\prime}\left(A, t_{i}, t_{0}, \tau\right)}}{N-n} \\
\text { where } \mu_{i}=\left\{\begin{array}{l}
\nu_{i}-\nu_{i-1}: \nu_{i}-\nu_{i-1} \geq \nu_{c r i t} \\
0: \nu_{i}-\nu_{i-1}<\nu_{c r i t}
\end{array}\right. \\
\text { and } \quad \nu_{i}=\frac{\sum_{j=i}^{i+n-1} D_{j}}{n} .
\end{gathered}
$$

Calculation of steppiness for a particular pulse proceeds as follows: each digitised point $\left(D_{j}\right)$ is converted to an average over $n$ points starting from $D_{i}$ and ending at $D_{i+n-1}$. $\quad N$ digitisation points are used from the start of the pulse $\left(t_{0}\right)$ to the end of the digitisation range. It was found that $n=3$ (corresponding to a 30 ns time window) gives optimum results. This value is labelled $\nu . \mu$ is the change in $\nu$ from one digitisation point to the next. If the change in $\nu$ is less than that for the arrival of 
2 photoelectrons $\left(\nu_{\text {crit }}\right)$, calculated based on a single photoelectron calibration of the PMT, then $\mu$ is set to zero. This was done because steps of one photoelectron are to be expected throughout the pulse and such a step is not an indication of pulse quality. The sum $\mu$ at each digitisation point divided by the gradient of the fit at the same point is calculated and converted to an average to acquire the value of steppiness. The gradient is used as a measure of how reasonable it is to have a step of certain size at each point and serves to weight the values.

The steppiness quantity and the full $\chi^{2}$ quantity were found to best identify noise events which have been fitted with a long time constant $(>300 \mathrm{~ns})$ whereas the partial $\chi^{2}$ quantities were found to work best on faster events ( $\left.<300 \mathrm{~ns}\right)$. Together, it has been found that these quantities may be used to efficiently identify the full range of noise events with time constants in the scintillation region. This is illustrated in Figure 5. Here the effect of the quality cuts on a sample of pure PMT coincident noise data is shown for events taken without scintillator, and for pulse heights equivalent to 4-6 $\mathrm{keV}$. It can be seen that the noise suppression factor is $>1000$ above $70 \mathrm{~ns}$ and still a factor 100 at lower time constants. Figure [6] shows the effect of quality cuts on real experimental data (lower points) compared with the effect of using conventional asymmetry cuts (upper points). An excellent reduction in noise can be seen in the critical scintillation-like region of 30-200 ns. The improvement at $100 \mathrm{~ns}$ being about a factor of 20. For $\mathrm{NaI}$ dark matter searches the key region of interest in a plot such as this is the left side of the main peak at 100-200 ns, the region expected for nuclear recoil events. The remarkable noise reduction and clarification of the electron signal here can be expected to improve sensitivity and control of systematic errors that result from usual noise subtraction techniques.

In addition, calculations were made of $\chi^{2}$ over what is expected to be the flat region of the pulse at $\left(t-t_{0}\right)>500 \mathrm{~ns}$ (see Figure [3). This quantity was found to be very effective for identifying events containing either PMT after-pulse signals or noise events within scintillation events. These events are rejected from NAIAD because the presence of the after-pulse may affect the accuracy of the least squares fitting routine.

Any loss of genuine scintillation or Cherenkov events from experimental data due to application of noise cuts clearly reduces experiment sensitivity. In the case of scintillators such as NaI using pulse shape analysis the important parameters are the proportion of electron recoils lost due to cuts and, for dark matter, the propotion of nuclear recoil events lost. Various test runs were performed to examine these efficiency losses using gamma and neutron sources. In the current apparatus, due to the saturation of the DAq at high rates, the ${ }^{60}$ Co calibration data has a much lower PMT noise content than normal low background data. The scintillation event rate for such data is much higher (typically $50 \mathrm{~s}^{-1}$ within the energy range $4-15 \mathrm{keV}$ compared to less than $1 \mathrm{~s}^{-1}$ for data collected with no source) but the PMT noise rate remains constant. The fraction of events cut from such data therefore gives a good indication of the efficiency of the cuts. Table 1 shows the efficiency of the newly developed cuts calculated in this manner compared to the efficiency of the traditional asymmetry cuts calculated by applying the cuts to ${ }^{60} \mathrm{Co}$ calibration data collected in the Boulby mine underground laboratory at a controlled crystal temperature of $8^{\circ} \mathrm{C}$. The quality cuts have been optimised to remove all noise with time constants in and around the scintillation region. Comparing, for instance, the energy band at $6-8 \mathrm{keV}$ (critical in dark matter searches) the loss of valid events due to asymmetry cuts is no greater than $16 \%$. The quality cut process 
yields a slightly smaller loss, lower by typically $4 \%$.

For NaI dark matter experiments the goal is to search for nuclear recoil events in experimental data, so efficiency in terms of fraction of rejected nuclear recoil events is also important. Neutron source calibration data collected on the surface has a very low noise content in much the same way as ${ }^{60}$ Co calibration data. Such data were used to measure the efficiency of the cuts. Table 2 compares cut efficiencies calculated by applying the cuts to ${ }^{60} \mathrm{Co}$ calibration data collected on the surface at room temperature. Table 3 compares cut efficiency calculated by applying the cuts to neutron calibration data collected on the surface at room temperature. These two tables show that the efficiencies for electron recoil events are similar to those for nuclear recoil events. Again the quality cuts cause slightly less loss of events, by typically $4-7 \%$.

\section{Conclusions}

An experiment to acquire a sample of pure PMT coincident noise data was performed using a low background shielding array underground at the Boulby facility. These data were analysed and parameters developed to distinguish and thereby cut this noise from real data. The newly developed cuts have been shown to be more effective at removing noise from experimental data and more efficient in preserving scintillation events compared to the asymmetry cuts currently used. In the typical scintillator region of around 100 ns the noise rejection is improved by a factor of 20. These cuts have further been shown to preserve nuclear recoil scintillation events with the same efficiency as electron recoil scintillation events and in both cases with slightly better efficiency than possible with assymetry cuts (by about 4-7\%). Similar quality cuts may prove effective in a range of experiments using PMTs required to work near threshold. Furthermore, since the new technique no longer relies on measuring coincidence asymmetry, it can in principle be applied to single PMT set-ups. The cuts developed here will be used in analysing data from NAIAD and are expected to produce a significant improvement in the sensitivity of the experiment and control of systematic error that previously resulted from noise subtraction.

\section{Acknowledgements}

The authors would like to thank the members of the UK Dark Matter Collaboration for their valuable assistance and advice. We are grateful to the Particle Physics and Astronomy Research Council for financial support and to Cleveland Potash Limited for their assistance. M. Robinson would also like to thank Hilger Crystals for their support of his $\mathrm{PhD}$ work.

\section{References}

[1] H. R. Krall. IEEE Trans. Nucl. Sci., NS-14 (1967) 455.

[2] Burle Industries Photomultiplier Handbook, (1980) 56.

[3] A. W. P. Poon arXiv:nucl-ex/0110005, (2001). 
[4] J. J. Quenby et al. Physics Letters B, 351 (1995) 70.

[5] M. Robinson, PhD Thesis, The NAIAD dark matter experiment: backgrounds and analysis, University of Sheffield (2003).

[6] N. J. C. Spooner et al. Physics Letters B, 473 (2000) 330.

[7] V. A. Kudryavtsev et al. Astroparticle Physics, 17 (2002) 401.

[8] R. Lüscher et al. Nuclear Physics B (Proc. Suppl.), 95 (2001) 233.

[9] H. Park et al. Nuclear Instruments and Methods in Physics Research A, 491 (2002) 460.

[10] H. J. Kim et al. Nuclear Instruments and Methods in Physics Research A, 457 (2002) 471.

[11] H. T. Wong et al. Physics Letters B, 536 (2002) 203.

[12] B. Ahmed at al. Astroparticle Physics, 17 (2003) 691. 
Table 1: Effect of cuts on scintillation events in underground ${ }^{60} \mathrm{Co}$ calibration data

\begin{tabular}{|c|c|c|c|}
\hline $\begin{array}{c}\text { energy range } \\
\text { (keV) }\end{array}$ & $\begin{array}{c}\text { without cuts } \\
\text { (events) }\end{array}$ & $\begin{array}{c}\text { quality cuts } \\
\text { (events remaining) }\end{array}$ & $\begin{array}{c}\text { asymmetry cuts } \\
\text { (events remaining) }\end{array}$ \\
\hline $4-6$ & 1076 & $911(85 \%)$ & $834(78 \%)$ \\
$6-8$ & 1081 & $958(88 \%)$ & $910(84 \%)$ \\
$8-10$ & 1106 & $1069(97 \%)$ & $1010(91 \%)$ \\
$10-12$ & 1081 & $1062(98 \%)$ & $1018(94 \%)$ \\
\hline
\end{tabular}

Table 2: Effect of cuts on scintillation events in surface ${ }^{60} \mathrm{Co}$ calibration data

\begin{tabular}{|c|c|c|c|}
\hline $\begin{array}{c}\text { energy range } \\
\text { (keV) }\end{array}$ & $\begin{array}{c}\text { without cuts } \\
\text { (events) }\end{array}$ & $\begin{array}{c}\text { quality cuts } \\
\text { (events remaining) }\end{array}$ & $\begin{array}{c}\text { asymmetry cuts } \\
\text { (events remaining) }\end{array}$ \\
\hline $4-6$ & 1521 & $1240(82 \%)$ & $1209(79 \%)$ \\
$6-8$ & 3872 & $3619(93 \%)$ & $3380(87 \%)$ \\
$8-10$ & 7074 & $7024(99 \%)$ & $6532(92 \%)$ \\
$10-12$ & 10442 & $10382(99 \%)$ & $9918(95 \%)$ \\
\hline
\end{tabular}

Table 3: Effect of cuts on scintillation events in surface neutron calibration data

\begin{tabular}{|c|c|c|c|}
\hline $\begin{array}{c}\text { energy range } \\
\text { (keV) }\end{array}$ & $\begin{array}{c}\text { without cuts } \\
\text { (events) }\end{array}$ & $\begin{array}{c}\text { quality cuts } \\
\text { (events remaining) }\end{array}$ & $\begin{array}{c}\text { asymmetry cuts } \\
\text { (events remaining) }\end{array}$ \\
\hline $4-6$ & 6488 & $5458(84 \%)$ & $5234(81 \%)$ \\
$6-8$ & 13179 & $12477(95 \%)$ & $11887(90 \%)$ \\
$8-10$ & 20554 & $20413(99 \%)$ & $19359(94 \%)$ \\
$10-12$ & 27019 & $26876(99 \%)$ & $26097(97 \%)$ \\
\hline
\end{tabular}




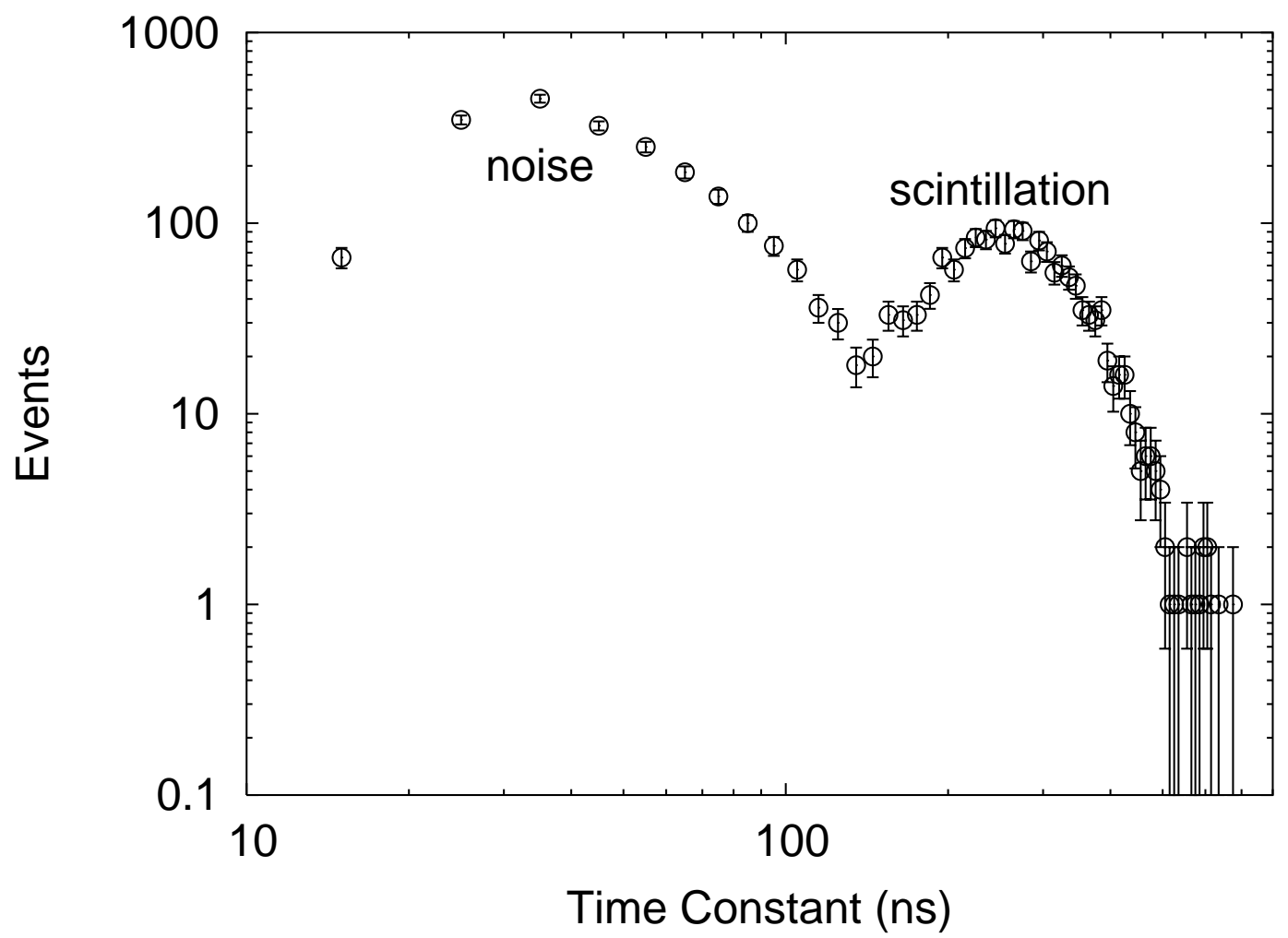

Figure 1: Typical NAIAD time constant distribution for the energy range 4-6 keV showing the encroachment of the noise into the scintillation region in data selected by asymmetry cuts

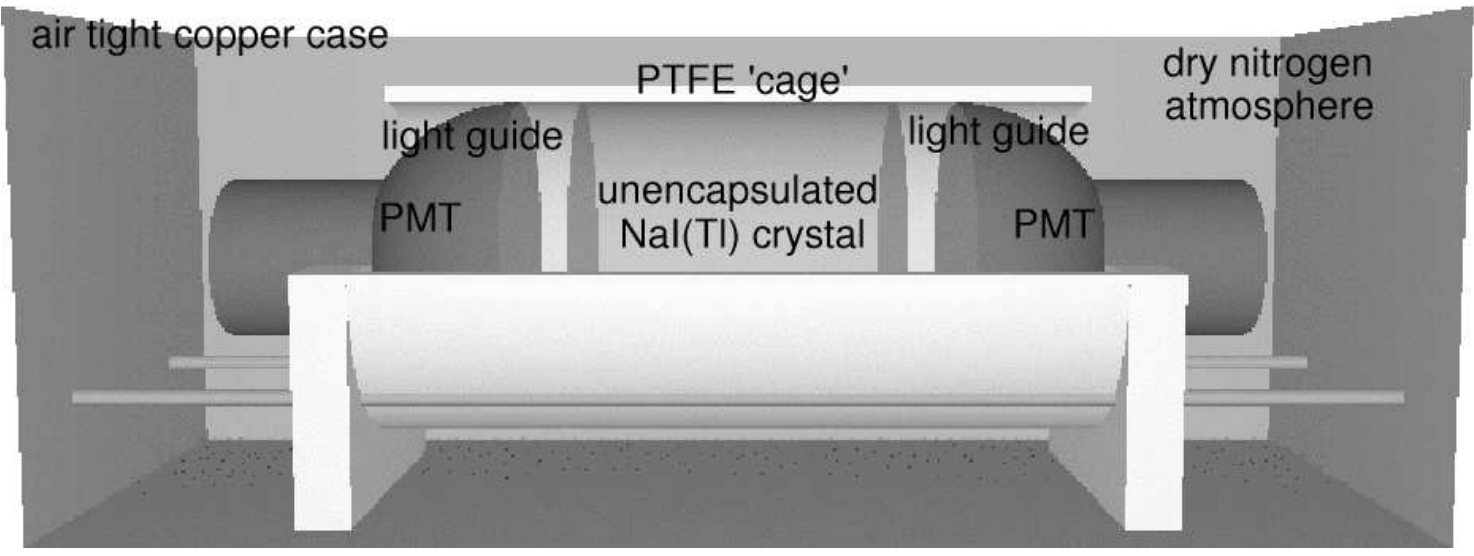

Figure 2: Diagram of standard NAIAD detector module 


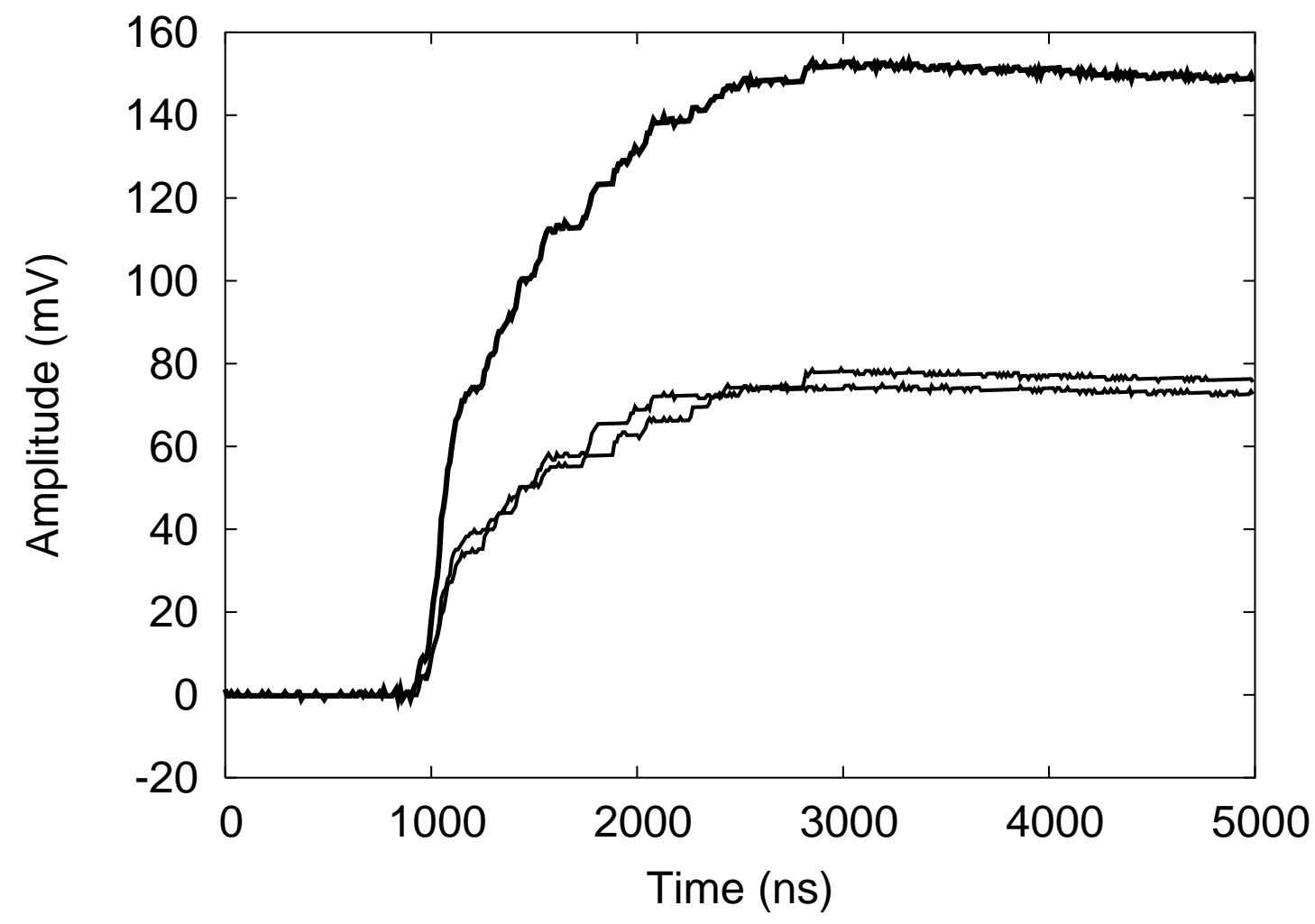

Figure 3: Example scintillation event from NAIAD. The 2 lower curves show the integrated signals from the individual PMTs. The upper curve shows the sum of these signals. 


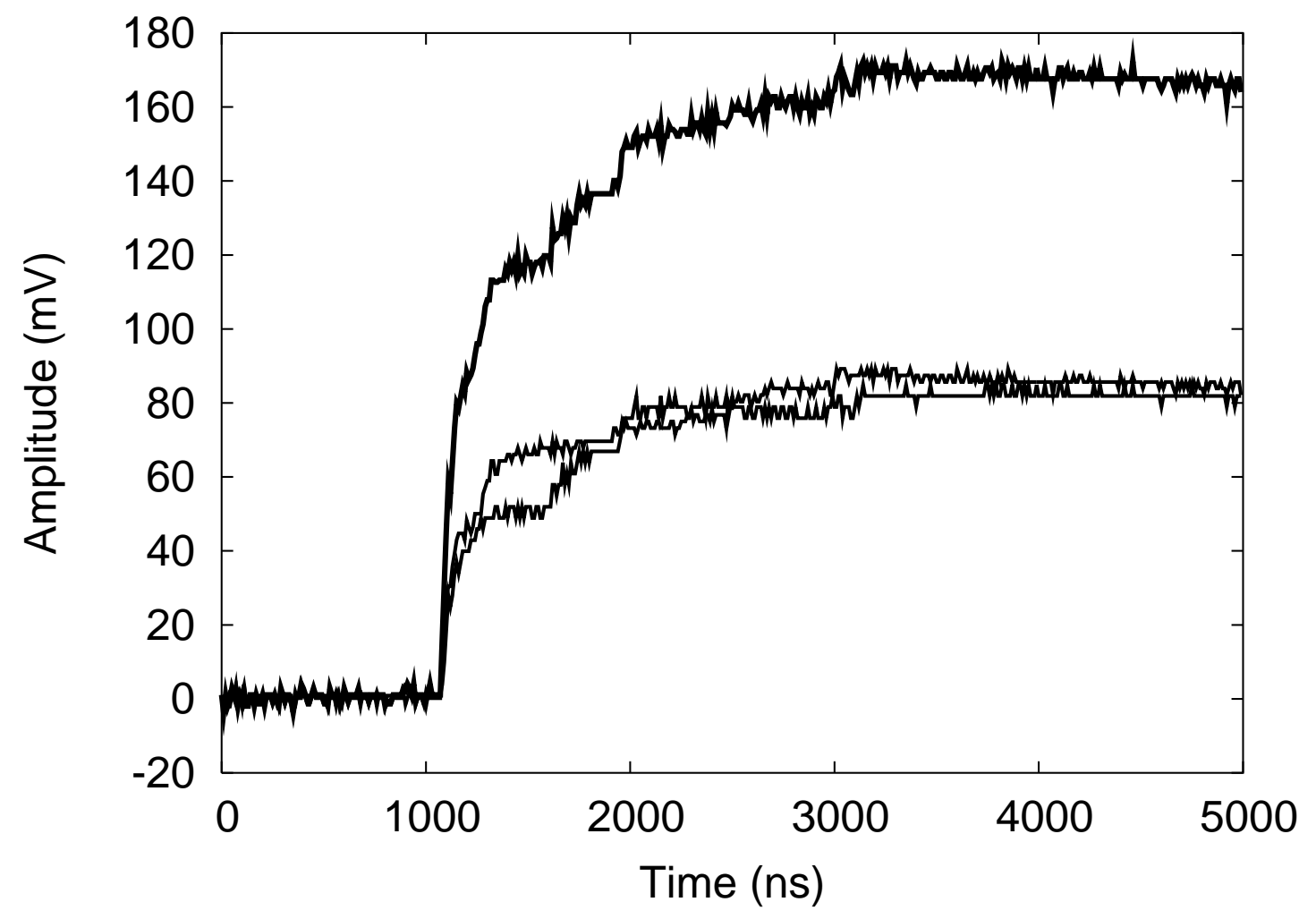

Figure 4: Event collected in noise measurement experiment mimicking scintillation. As in Figure 3 the 2 lower curves show the integrated signals from the individual PMTs. The upper curve shows the sum of these signals. 


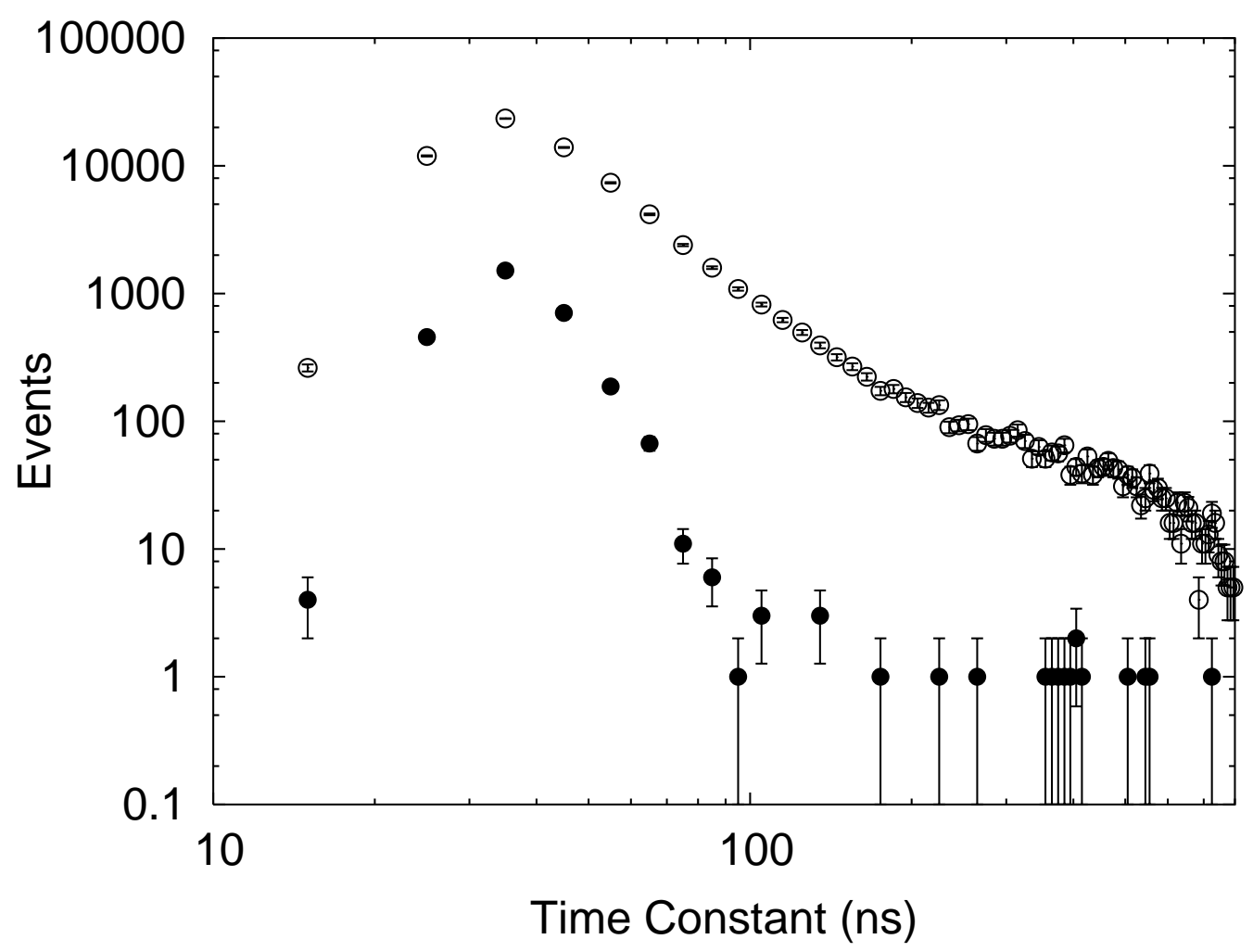

Figure 5: Time constant distribution from noise experiment data corresponding to the energy range 4-6 keV; open circles represent uncut data, filled circles represent quality cut data 


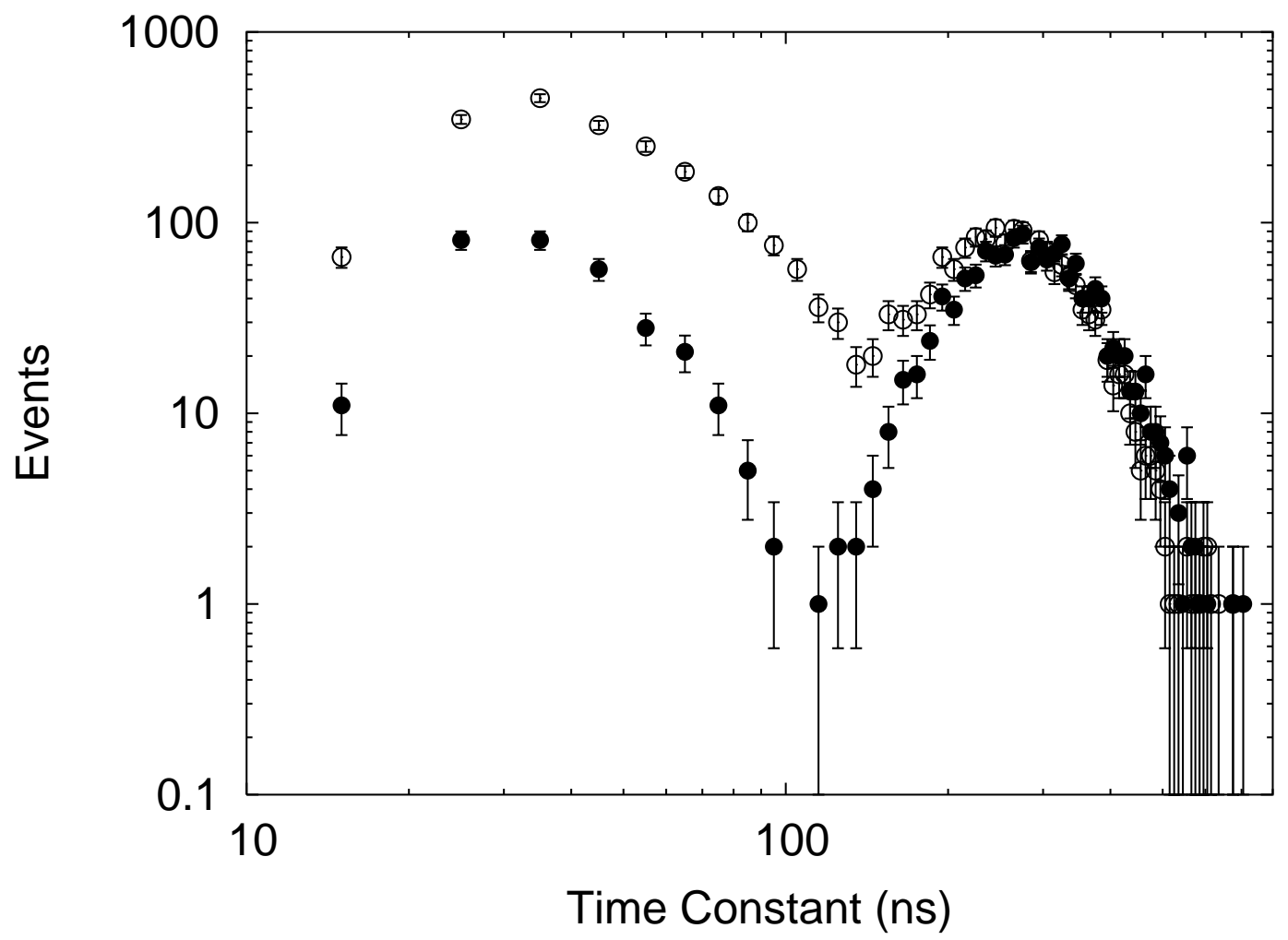

Figure 6: Time constant distribution showing relative effectiveness of quality cuts (filled circles) versus traditional cuts (hollow circles) in the energy range 4-6 keV 